\title{
UNIVERSAL FUNCTIONS IN SEVERAL COMPLEX VARIABLES
}

\author{
P. S. CHEE \\ (Received 25 May 1978; revised 6 March 1979) \\ Communicated by E. Strzelecki
}

\begin{abstract}
It is proved that there exists a universal good inner function in the open unit polydisc $U^{n}$, that is its nonEuclidean translates are dense in the closed unit ball of $H^{\infty}\left(U^{n}\right)$ and that there exists a universal function in the open unit ball $B_{n}$ of $\mathbf{C}^{n}$. These generalize Heins' result on universal Blaschke products.
\end{abstract}

1980 Mathematics subject classification (Amer. Math. Soc.): primary 32 A 10.

Keywords and phrases: Polydisc, ball, holomorphic automorphism, bounded holomorphic function, universal Blaschke product, universal inner function.

\section{Introduction}

Let $\Omega$ be a region in the space $\mathbf{C}^{n}$ of $n$ complex variables, $\{\Phi\}$ a family of holomorphic automorphisms of $\Omega$ and $\mathscr{F}$ a family of holomorphic functions in $\Omega$. A function $g \in \mathscr{F}$ is called a universal function of $\mathscr{F}$ relative to $\{\Phi\}$ if for any $f \in \mathscr{F}$, there exists a sequence $\left\{\Phi_{k}\right\}_{1}^{\infty}$ from $\{\Phi\}$ such that

$$
g \circ \Phi_{k} \rightarrow f
$$

uniformly on compact subsets of $\Omega$.

G. D. Birkhoff (1929) was the first to obtain a universal function when he proved that in $\mathbf{C}$ there exists a universal entire function relative to translations of the form $z \rightarrow z+a, a \in \mathbf{R}$. This was later generalized by Seidel and Walsh (1941) to any simply-connected region instead of $\mathbf{C}$.

Heins (1955) considered universal functions in the family $\mathscr{B}$ of holomorphic functions in the open unit disc $U$ bounded by 1 and obtained the following. 
HEINS' THEOREM. There exist $a$ Blaschke product $b$ and a monotone increasing sequence $\left\{x_{k}\right\}$ with $x_{0}=0$ and $\lim x_{k}=1$ such that

(i) the point $z=1$ is the sole cluster point of the zeros of $b$,

(ii) to every $f \in \mathscr{B}$ corresponds a subsequence $\left\{x_{k(r)}\right\}$ for which

$$
b\left(\frac{z+x_{k(r)}}{1+x_{k(r)} z}\right) \rightarrow f(z)
$$

uniformly on compact subsets of $U$.

He called $b$ a universal Blaschke product.

For several complex variables, the results of Birkhoff and Seidel and Walsh hold true with little change in the proofs. The purpose of this paper is to consider analogues of Heins' theorem in the open unit polydisc $U^{n}$ and in the open unit ball $B_{n}$ of $C^{n}$ respectively. We show that (Theorem 1) there exists a universal good inner function in $U^{n}$ and that (Theorem 2) there exists a universal function in $B_{n}$. However, in the case of $B_{n}$, we were not able to show that the universal function can be chosen to be inner.

This is part of the author's thesis at the University of Wisconsin. I wish to thank Professor W. Rudin for helpful advice during the preparation of this work.

\section{Preliminaries}

Points of $\mathbf{C}^{n}$ will be denoted by $z=\left(z_{1}, \ldots, z_{n}\right)$ where $z_{j} \in \mathbf{C}, 1 \leqslant j \leqslant n$. The open unit polydisc $U^{n}$ and the open unit ball $B_{n}$ are defined by

$$
\begin{aligned}
& U^{n}=\left\{z \in \mathbf{C}^{n}:\left|z_{j}\right|<1,1 \leqslant j \leqslant n\right\}, \\
& B_{n}=\left\{z \in \mathbf{C}^{n}:\left|z_{1}\right|^{2}+\ldots+\left|z_{n}\right|^{2}<1\right\} .
\end{aligned}
$$

The distinguished boundary of $U^{n}$ is the $n$-dimensional torus

$$
T^{n}=\left\{z \in \mathbf{C}^{n}:\left|z_{j}\right|=1,1 \leqslant j \leqslant n\right\} .
$$

For an open subset $\Omega$ of $\mathbf{C}^{n}$, let $H(\Omega)$ denote the set of all holomorphic functions in $\Omega$ and $H^{\infty}(\Omega)$ the set of all bounded ones. Further, let

$$
\begin{aligned}
& A(\Omega)=\left\{f \in H^{\infty}(\Omega): f \text { is continuous on } \bar{\Omega}\right\}, \\
& \mathscr{B}(\Omega)=\left\{f \in H^{\infty}(\Omega):\|f\|_{\infty} \leqslant 1\right\} .
\end{aligned}
$$

We let $H(\Omega)$ or its subsets have the compact-open topology so that convergence means uniform convergence on compact subsets of $\Omega$.

A function $f \in H^{\infty}\left(U^{n}\right)$ is an inner function in $U^{n}$ if $\lim _{r \rightarrow 1} f(r z)=f^{*}(z)$ has absolute value 1 for almost all $z \in T^{n}$ (relative to the Haar measure $m_{n}$ on $T^{n}$ ). An 
inner function $f$ in $U^{n}$ is said to be good if

$$
\lim _{r \rightarrow 1} \int_{T^{n}} \log |f(r w)| d m_{n}(w)=0 .
$$

When $n=1$, the good inner functions are precisely the Blaschke products. (See Rudin (1969).)

A subset $E$ of $\Omega$ is a uniqueness set (or $D$-set) for a family $\mathscr{F} \subseteq H(\Omega)$ if $f \in \mathscr{F}$ and $f=0$ on $E$ imply $f=0$ in $\Omega$.

The following version of Vitali's theorem will be useful.

VITALI'S THEOREM. Let the sequence $\left\{f_{k}\right\}$ form a normal family in a region $\Omega$ in $\mathbf{C}^{n}, E$ a uniqueness set for $\overline{\mathscr{F}}$, the closure of $\mathscr{F}$ in the compact-open topology. If $f_{k}$ converges pointwise on $E$, then $f_{k}$ converges uniformly on compact subsets of $\Omega$.

For a proof in one variable, see for example Markushevich (1965) Section 87. The same proof works for several variables.

\section{Universal inner function in $U^{n}$}

Let $x_{k}=\left(x_{k 1}, \ldots, x_{k n}\right) \in \mathbf{R}^{n}, 0 \leqslant x_{k j}<1,1 \leqslant j \leqslant n$. Let

and

$$
\varphi_{k j}\left(z_{j}\right)=\frac{z_{j}+x_{k j}}{1+x_{k j} z_{j}}, \quad 1 \leqslant j \leqslant n
$$

$$
\Phi_{k}(z)=\left(\varphi_{k 1}\left(z_{1}\right), \ldots, \varphi_{k n}\left(z_{n}\right)\right), \quad z \in U^{n} .
$$

Then each $\Phi_{k}$ is a holomorphic automorphism of $U^{n}$, and is continuous on $\bar{U}^{n}$. In this section, we show that there exists a universal good inner function in $U^{n}$ relative to the automorphisms $\left\{\Phi_{k}\right\}$.

THEOREM 1. There exist a good inner function $g$ in $U^{n}$ and a sequence $\left\{x_{k}\right\}$ in $\mathbf{R}^{n}$ with $0 \leqslant x_{k j}<1$ and $\lim _{k \rightarrow \infty} x_{k j}=1,1 \leqslant j \leqslant n$, with the property that to every $f \in \mathscr{B}=\mathscr{B}\left(U^{n}\right)$ corresponds a subsequence $\left\{x_{k(r)}\right\}$ such that

$$
g \circ \Phi_{k(r)} \rightarrow f
$$

uniformly on compact subsets of $U^{n}$, where $\Phi^{k}$ is obtained from $x_{k}$ as given by (1).

The following result of Rudin (1969), Theorem 5.5.1, is needed for the proof.

LEMma 2. There exists a countable set $\left\{A_{k}\right\}_{1}^{\infty}$ which is dense in $\mathscr{B}$ in the compactopen topology, where $A_{k} \in A\left(U^{n}\right),\left|A_{k}(z)\right|=1$ for all $z \in T^{n}$. 
For our purposes, we need to modify the functions $A_{k}$ in Lemma 2 so that they have the value 1 at the points $\pm(1, \ldots, 1)$. To that end, let $c \in U, \eta_{1}, \eta_{2} \in T$ be given. Fix an $n$th root of each of these and denote them by $c^{1 / n}, \eta_{1}^{1 / n}, \eta_{2}^{1 / n}$ respectively. It was shown in Heins (1955) that there exists $\varphi: \bar{U} \rightarrow \bar{U}$, which is holomorphic in $U$ and

$$
\varphi(0)=c^{1 / n}, \varphi(1)=\eta_{1}^{1 / n}, \quad \varphi(-1)=\eta_{2}^{1 / n} .
$$

For $z \in \bar{U}^{n}$, define $\Psi(z)=\varphi\left(z_{1}\right) \ldots \varphi\left(z_{n}\right)$. Then

$$
\Psi(0)=c, \quad \Psi(1, \ldots, 1)=\eta_{1}, \quad \Psi(-1, \ldots,-1)=\eta_{2} .
$$

With $A_{k}$ as in Lemma 2, we define $G_{k}=A_{k} \Psi_{k}$, where $\Psi_{k}$ is of the kind just described, with $\Psi_{k}(0)=1-2^{-k}$,

$$
\Psi_{k}(1, \ldots, 1)=\frac{1}{A_{k}(1, \ldots, 1)}, \quad \Psi_{k}(-1, \ldots,-1)=\frac{1}{A_{k}(-1, \ldots,-1)} .
$$

Since $\left\|\Psi_{k}\right\|_{\infty} \leqslant 1$ for all $k,\left\{\Psi_{k}\right\}$ is a normal family in $U^{n}$. Since $\Psi_{k}(0)=1-2^{k}$, the maximum modulus theorem implies that any convergent subsequence of $\left\{\Psi_{k}\right\}$ has the constant limit 1 in $U^{n}$. Since $\left\{A_{k}\right\}$ is dense in $\mathscr{B}$, any $f \in \mathscr{B}$ is the limit of a convergent subsequence $\left\{\boldsymbol{A}_{\boldsymbol{k}(r)}\right\}$. Since the corresponding sequence $\left\{\Psi_{k(r)}\right\}$ is a normal family, there exists a convergent subsequence $\left\{\Psi_{k\left(r_{j}\right)}\right\}$, with limit 1 in $U^{n}$. Then the sequence $\left\{G_{k\left(r_{j}\right)}\right\}$ converges to $f$. Hence $\left\{G_{k}\right\}$ is dense in $\mathscr{B}$. Further, $G_{k}(1, \ldots, 1)=G_{k}(-1, \ldots,-1)=1$.

Now let

and

$$
\begin{gathered}
x_{k} \in \mathbf{R}^{n}, \quad 0 \leqslant x_{k j}<1, \quad 1 \leqslant j \leqslant n, \\
I_{k}=\left\{\left(y_{1}, \ldots, y_{n}\right) \in \mathbf{R}^{n}:-1 \leqslant y_{j} \leqslant x_{k j}, \quad 1 \leqslant j \leqslant n\right\}
\end{gathered}
$$

$$
V_{k}=\left\{z \in U:|1-z|<2^{-k}\right\} .
$$

Let $\Phi_{k}$ be the automorphism associated with $x_{k}$ as described by (1). Let $g_{k}=G_{k} \circ \Phi_{k}^{-1}$. Then the real numbers $x_{k j}$ can be chosen so that $x_{k j} \rightarrow 1$ and

(i) $g_{k+1}\left(I_{k}\right) \subseteq V_{k+1}$,

(ii) $g_{r}\left(x_{k+1}\right) \in V_{k+1}, r=1,2, \ldots, k$.

This is a consequence of the fact that $G_{k}(1, \ldots, 1)=G_{k}(-1, \ldots,-1)=1$ and that each $G_{k}$ is continuous on $\bar{U}^{n}$.

With $g_{k}$ chosen as above, we define $g(z)=\prod_{1}^{\infty} g_{k}(z)$. Then $g$ satisfies the requirements of Theorem 1. The infinite product converges on $I_{1}=\bigcap_{1}^{\infty} I_{k}$ by (i). Since $I_{1}$ is a uniqueness set for $H^{\infty}\left(U^{n}\right)$, the product converges uniformly on compact subsets of $U^{n}$, by Vitali's theorem. Since each factor $g_{k}$ is a good inner function in $U^{n}$, so is $g$ (see Rudin (1969), p. 118).

It remains to show that $\left\{g \circ \Phi_{k}\right\}$ is dense in $\mathscr{B}$. Since $\left\{G_{k}\right\}$ is dense in $\mathscr{B}$, it is enough to show that

$$
\lim _{k \rightarrow \infty}\left(G_{k}-g \circ \Phi_{k}\right)=0
$$


But $G_{k}-g \circ \Phi_{k}=G_{k}\left(1-\Pi_{j \neq k} g_{j} \circ \Phi_{k}\right)$. Since the product is in $\mathscr{B}$, (2) is a consequence of

$$
\lim _{k \rightarrow \infty} \prod_{j \neq k}\left(g_{j} \circ \Phi_{k}\right)(0)=1 .
$$

Since $\Phi_{k}(0)=x_{k}$, it suffices to show that

$$
\lim _{k \rightarrow \infty} \prod_{j \neq k} g_{j}\left(x_{k}\right)=1 .
$$

Let $g_{j}\left(x_{k}\right)=1+u_{j}(k)$. Then by (i) and (ii),

$$
\left|u_{j}(k)\right|<2^{-k} \text { if } j<k \text { and }\left|u_{j}(k)\right|<2^{-j} \text { if } j>k \text {. }
$$

Hence

$$
\begin{aligned}
\left|\prod_{j \neq k} g_{j}\left(x_{k}\right)-1\right| & \leqslant \prod_{j \neq k}\left(1+\left|u_{j}\right|\right)-1 \\
& \leqslant\left(1+2^{-k}\right)^{k-1} \prod_{j=k+1}^{\infty}\left(1+2^{-j}\right)-1 \\
& \rightarrow 0 \text { as } k \rightarrow \infty .
\end{aligned}
$$

\section{Universal functions in $B_{n}$}

The open unit ball $B_{n}$ has the following family of automorphisms. For each positive integer $k$, let $x_{k}$ and $y_{k}$ be nonnegative real numbers satisfying $x_{k}^{2}+y_{k}^{2}=1$. Let

$$
\Phi_{k}(z)=\left(\varphi_{k 1}(z), \varphi_{k 2}(z), \ldots, \varphi_{k n}(z)\right), \quad z \in B_{n}
$$

where

$$
\begin{aligned}
\varphi_{k 1}(z) & =\frac{z_{1}+x_{k}}{1+x_{k} z_{1}}, \\
\varphi_{k j}(z) & =\frac{y_{k} z_{j}}{1+x_{k} z_{1}}, \quad 2 \leqslant j \leqslant n .
\end{aligned}
$$

Each $\Phi_{k}$ is an automorphism of $\bar{B}_{n}$, with the inverse obtained from the above by replacing $x_{k}$ by $-x_{k}$.

THEOREM 3. There exists a universal function in $\mathscr{B}\left(B_{n}\right)$ relative to the automorphisms $\left\{\Phi_{k}\right\}$ gjven by (3).

The proof of Theorem 3 is exactly parallel to that of Theorem 1, the main difficulty being an analogue of Lemma 2 . We give only the proof of Lemma 4 below and omit the rest of the proof of Theorem 3 . 
LEMMA 4. There exists a sequence $\left\{A_{k}\right\}$ which is dense in $\mathscr{B}=\mathscr{B}\left(B_{n}\right)$, where $A_{k} \in A\left(B_{n}\right), A_{k} \in \mathscr{B}$, and

$$
\left|A_{k}( \pm 1,0, \ldots, 0)\right|=1 \text {. }
$$

Proof. We use a method discussed in Curtis (1969). As in the proof of Lemma 2, it is sufficient to show that given a polynomial $P$ with $|P(z)| \leqslant r<1$ for all $z \in \bar{B}_{n}$, a compact subset $K$ of $B_{n}$ and an $\varepsilon>0$, there exists an $f \in A\left(B_{n}\right) \cap \mathscr{B}$ with $|f( \pm 1,0, \ldots, 0)|=1$ and $|P-f|<\varepsilon$ on $K$. Fix such a compact set $K$.

Let

$$
M=\frac{4}{1-r}, \quad \eta=\frac{1+r}{2} \text { and } S=\left\{z \in \bar{B}_{n}:\left|z_{1}\right|=1\right\}
$$

For any positive integer $m$, let

$$
g_{m}(z)=\frac{P(z)+z_{1}^{2 m}}{1+z_{1}^{2 m} Q(z)}, \quad z \in \bar{B}_{n},
$$

where $\overline{Q(z)}=P\left(1 / \bar{z}_{1}, \bar{z}_{2}, \ldots, \bar{z}_{n}\right)$. Then $g_{m}$ is in $A\left(B_{n}\right)$ for sufficiently large $m$, and for all $m$,

$$
\left|g_{m}(z)\right|=1 \text { for all } z \in S
$$

since $z \in S$ implies $z=\left(z_{1}, 0, \ldots, 0\right)$ with $\left|z_{1}\right|=1$. In particular, $g_{m}( \pm 1,0, \ldots, 0)$ is independent of $m$ and has absolute value 1 . Note that since $\left|z_{1}\right|<1$ on $K$,

$$
\left\|g_{m}-P\right\|_{K} \rightarrow 0 \text { as } m \rightarrow \infty \text {. }
$$

Furthermore, there exists an $m_{0}>0$ such that

$$
\left|g_{m}\right| \leqslant M \text { on } \bar{B}_{n} \text { for all } m \geqslant m_{0},
$$

and given any compact subset $F$ of $\bar{B}_{n}$ with $F \cap S=\varnothing$, there exists an $m_{F}$ such that $\left|g_{m}\right| \leqslant \eta$ on $F$, for all $m \geqslant m_{F}$.

Let $0<r_{1}<r_{2}<\ldots<1, r_{k} \rightarrow 1$. Let

$$
V_{k}=\left\{z \in \bar{B}_{n}:\left|z_{1}\right|>r_{k}\right\} \text {. }
$$

Choose $r_{1}$ such that $V_{1} \cap K=\varnothing$. Choose $a$ such that

Let

$$
0<a<1 \text { and } a M+(1-a) \eta<1 \text {. }
$$

$$
\varepsilon_{k}=(1-a)^{k}(1-a M-(1-a) \eta) .
$$

Choose $m_{1}>m_{0}$ such that $\left\|g_{m_{1}}-P\right\|_{K}<\varepsilon$, and

$$
\left|g_{m_{1}}(z)\right| \leqslant \eta, \quad z \in F_{0},
$$

where $F_{0}$ is a compact set containing $K$ with $F_{0} \cap S=\varnothing$. Let $f_{1}=g_{m_{1}}$. Define

$$
W_{1}=V_{1} \cap\left\{z \in \bar{B}_{n}: \| a f_{1}(z)|-a|<\varepsilon_{1}\right\}
$$


and $F_{1}=\bar{B}_{n}-W_{1}$. By (4), $S \subseteq W_{1}$ and hence $F_{1} \cap S=\varnothing$. Now choose $m_{2}$ such that with $f_{2}=g_{m_{2}}$, we have

$$
\left|f_{2}(z)\right| \leqslant \eta, \quad z \in F_{1},
$$

and $\left\|f_{2}-P\right\|_{R}<\varepsilon$. Then $z \in W_{1}$ implies

Define

$$
a\left|f_{1}(z)\right|+a(1-a)\left|f_{2}(z)\right|<a+\varepsilon_{1}+a(1-a) M=1-(1-a)^{2} \eta .
$$

$$
W_{2}=V_{2} \cap\left\{z \in W_{1}:\left|\sum_{k=1}^{2} a(1-a)^{k-1}\right| f_{k}(z)\left|-\sum_{k=1}^{2} a(1-a)^{k-1}\right|<\varepsilon_{2}\right\},
$$

and $F_{2}=\bar{B}_{n}-W_{2}$. Then $F_{2} \cap S=\varnothing$.

Continuing inductively, we assume that open sets

$$
\bar{B}_{n}=W_{0} \supset W_{1} \supset W_{2} \supset \ldots \supset W_{k-1}
$$

and functions $f_{1}, \ldots, f_{k} \in A\left(B_{n}\right)$ have been chosen such that

$$
\begin{gathered}
\left|f_{j}(z)\right| \leqslant M \text { for } z \in \bar{B}_{n} ; \quad\left|f_{j}(z)\right|=1 \text { for } z \in S ; \\
\left\|f_{j}-P\right\|_{K}<\varepsilon ; \quad\left|f_{j}(z)\right| \leqslant \eta \text { for } z \in F_{j-1}=\bar{B}_{n}-W_{j-1}, \quad 1 \leqslant j \leqslant k,
\end{gathered}
$$

and $z \in W_{j-1}$ implies $\sum_{i=1}^{j} a(1-a)^{i-1}\left|f_{i}(z)\right| \leqslant 1-(1-a)^{j} \eta$. Now define

$$
W_{k}=V_{k} \cap\left\{z \in W_{k-1}:\left|\sum_{i=1}^{k} a(1-a)^{i-1}\right| f_{i}(z)\left|-\sum_{i=1}^{k} a(1-a)^{i-1}\right|<\varepsilon_{k}\right\}
$$

and $F_{k}=\bar{B}_{n}-W_{k}$. Then $F_{k} \cap S=\varnothing$.

Choose $m_{k+1}$ such that with $f_{k+1}=g_{m_{k+1}}$, we have

$$
\left|f_{k+1}(z)\right| \leqslant M, \quad z \in \bar{B}_{n} ; \quad\left|f_{k+1}(z)\right|=1, \quad z \in S ;
$$

and

$$
\left|f_{k+1}(z)\right| \leqslant \eta, \quad z \in F_{k}
$$

$$
\left\|f_{k+1}-P\right\|_{\mathrm{K}}<\varepsilon
$$

Then $z \in W_{k}$ implies

$$
\begin{aligned}
\sum_{i=1}^{k+1} a(1-a)^{i-1}\left|f_{i}(z)\right| & <\sum_{i=1}^{k} a(1-a)^{i-1}+\varepsilon_{k}+a(1-a)^{k} M \\
& =1-(1-a)^{k+1} \eta .
\end{aligned}
$$

With the sequence $\left\{f_{k}\right\}$ chosen as above, we define

Then

$$
f=\sum_{k=1}^{\infty} a(1-a)^{k-1} f_{k}
$$

$$
f \in A\left(B_{n}\right), \quad\|f-P\|_{K} \leqslant \sum_{k=1}^{\infty} a(1-a)^{k-1}\left\|f_{k}-P\right\|_{K}<\varepsilon
$$


and

$$
|f( \pm 1,0, \ldots, 0)|=1 \text {. }
$$

We claim that $f \in \mathscr{B}$. Let $z \in B_{n}$. Then there exists $k$ such that $z \in W_{k-1}-W_{k}$. So,

$$
\begin{aligned}
|f(z)| & \leqslant \sum_{i=1}^{k} a(1-a)^{i-1}\left|f_{i}(z)\right|+\sum_{i=k+1}^{\infty} a(1-a)^{i-1}\left|f_{i}(z)\right| \\
& <1-(1-a)^{k} \eta+(1-a)^{k} \eta=1 .
\end{aligned}
$$

This completes the proof of Lemma 4.

\section{References}

G. D. Birkhoff (1929), 'Demonstration d'un théorème élémentaire sur les fonctions entières', Comptes Rendus Acad. Sci. Paris 189, 473-475.

P. C. Curtis, Jr. (1969), 'Peak points for algebras of analytic functions', J. Functional Analysis 2, 35-47.

M. Heins (1955), 'A un ' rersal Blaschke product', Archiv der Math. 6, 41-44.

A. I. Markushevich (1965), Theory of functions of a complex variable, Vol. I (Prentice-Hall, Englewood Cliffs, iN.J.).

W. Rudin (1969), Function theory in polydiscs (W. A. Benjamin, New York).

W. Seidel and J. L. Walsh (1941), 'On approximation by Euclidean and non-Euclidean translations of an analytic function', Bull. Amer. Math. Soc. 47, 916-920.

Department of Mathematics

University of Malaya

Kuala Lumpur

Malaysia 